\title{
Exploitation of Bistatic Doppler Measurements in Multistatic Tracking
}

\author{
Thomas Lang \\ Air \& Naval Systems \\ General Dynamics Canada \\ Ottawa, Ontario, Canada. \\ tom.lang@gdcanada.com
}

\author{
Geoff Hayes \\ Air \& Naval Systems \\ General Dynamics Canada \\ Ottawa, Ontario, Canada. \\ geoff.hayes@gdcanada.com
}

\begin{abstract}
The exploitation of bistatic Doppler measurements for multistatic tracking is considered. It is found through simulation, that, while the velocity estimation of the standard extended Kalman filter is improved in monostatic situations and multistatic situations where measurement errors are small, a degradation in performance is observed in multistatic situations where the measurement errors are realistically large. A bistatic modification to the alternate EKF of Bizup and Brown [9] is proposed as a means of exploiting bistatic Doppler measurements in realistic multistatic situations. Simulation results confirm that the proposed filter outperforms the standard EKF and can produce significantly improved target velocity estimates when Doppler measurements are exploited in multistatic applications.
\end{abstract}

Keywords: Tracking, Kalman filtering, nonlinear state estimation, bistatic, multistatics, sonar.

\section{Introduction}

The maritime defense community is currently faced with several major challenges from underwater threats. On the one hand, continuing advances in quietening technologies, and the shift of the principal military threat from large, primarily nuclear-powered Soviet submarines with wellstudied acoustic signatures to a panoply of relatively small, quiet, diesel-powered submarines manufactured and deployed by a number of nations, have acted to increase the difficulty of detecting, localizing, tracking, and identifying submarine targets using traditional passive and active sonar methods. On the other hand, the peace dividend realized by the end of the Cold War has acted to reduce the budget of national defense departments, with the result that limited assets and manpower are generally available to fulfill the vital military objectives of area clearance and area denial in times of hostility. The combined influence of these factors has led to the current situation, in which traditional passive and active sonar methods are generally perceived as inadequate for achieving these very relevant military objectives.
The perceived inadequacy of traditional passive and active sonar methods to satisfy these objectives has led to a search for new solutions. While this search has been pursued in a number of different directions, including nonacoustic sensors as but one example, the direction we shall focus on in this paper is the deployment of multistatic active sonar operations, commonly called simply multistatics. In a conventional monostatic active sonar, an acoustic receive sensor (typically consisting of an array of one or more hydrophones) listens for reflections from targets ("echoes") of an energy transmission from an active acoustic source co-located with the receiver on a common platform. Such a platform may be, for example, a hull mounted sonar on a ship, or an active sonobuoy deployed from a maritime patrol aircraft, helicopter, or ship. In multistatics, by contrast, a number of non-colocated acoustic sensors simultaneously listen for target echoes arising from one or more non-co-located acoustic sources. Common examples of receivers are towed line arrays and passive sonobuoys; sources are hull mounted sonars, and active sonobuoy sources. The minimum multistatic configuration, consisting of a single non-colocated source and receiver, is referred to as the bistatic configuration. Indeed, in practice a multistatic field is commonly processed as a collection of bistatic source/receiver pairs. The main difficulty in employing just a single bistatic pair is that the reflectivity of real targets is usually highly aspect dependent, such that correct geometry between source, target, and receiver is critical in order to achieve a good probability of detecting the echo from the target. This ideal geometry is referred to as the "glint" geometry, and, among other things, depends strongly on the usually unknown, or poorly known, target heading. The fundamental advantage of multistatics is that, through the simultaneous deployment of multiple sensors, the probability of detecting a "glint" echo is multiplied. The promise held by multistatics is therefore that militarily useful volumes of ocean can be searched for modern threats in a reasonable amount of time using limited assets and manpower.

The basic idea behind multistatics is not new [1]. While the development of new low frequency active sources and receivers has contributed to the feasibility of modern multistatics, especially in the littoral, the key enabling 
technology has been the recent advances in the computational capabilities of shipborne and airborne acoustic processors. And while it is possible to conduct multistatics operations without cooperation between platforms, for example from a single maritime patrol aircraft, helicopter, or ship, there can be no doubt of the synergistic relationship between multistatics and the modern military concept of multi-platform network enabled operations.

Even with its many advantages, tracking a target in a multistatic context remains a challenging proposition. Glint echo detections are few and far between; any single receiver will probably not detect the target for longer than a small number of consecutive ping transmissions. The tracking problem presented by this low probability of detection is compounded in littoral operations by a large quantity of clutter (false alarms) that serves to obscure these few target echoes. Given these difficulties, clearly all available measurements should be exploited to maximize the performance of a multistatic tracker. In some multistatic processing systems, this includes observations of target Doppler (also known as radial velocity or range-rate).

A number of multistatic sonar trackers have been reported in the literature [2-6]. Coraluppi and Grimmett presented a design based on conversion of raw time-difference-ofarrival (TDOA) and bearing measurements to 2DCartesian fixes for input to a linear Kalman filter [2]. De Theije and his collaborators describe a tracker that initializes and updates single-sensor tracks in sensor coordinates, until a second sensor starts to contribute measurements to the track, at which time the track is converted to a more general state space and filtering is performed using an extended Kalman filter [3]. La Cour describes a Bayesian tracking approach in which the posterior distribution of the target state is represented by a random sample or "ensemble" of target states [4]. Willett and Coraluppi compared the tracking performance of three types of multistatic tracker, employing the basic Kalman formalism for state correction [5]. Hempel described a Probabilistic Multiple-Hypothesis Tracker (PMHT) that exploits observed target amplitude as well as TDOA and bearing observations [6]. None of the reported trackers appears to exploit bistatic Doppler measurements.

On the other hand, the exploitation of range-rate measurements from monostatic radar has been investigated by a number of researchers, including [7-13]. The significant improvements in radar tracking performance available when range-rate measurements are exploited were described in their book by Farina and Studer [7]. Kameda, Tsujimichi, and Kosuge describe how range-rate measurements may be exploited in a JPDAF tracker to improve data association performance in a dense target environment [8]. Bizup and Brown propose an alternate extended Kalman filter (AEKF) to mitigate the damaging effects of the large nonlinearities inherent in tracking using range-rate observations in Cartesian state space [9]. Bar-Shalom points out that further performance gains are possible by accounting in the tracking system for the correlation between range and range rate measurement errors arising from the use of certain pulse types [10]. Wang, He and Long propose a modified EKF for accounting for these correlations [11]. Additional methods for incorporating these correlations in the tracker design are proposed by Duan and collaborators $[12,13]$. The potential advantages of exploiting bistatic Doppler observations in multistatic sonar tracking are presented by Grimmett [14].

The major contribution of this paper is the first recorded application, known to the authors, of bistatic Doppler measurements to multistatic sonar tracking. Further, this is the first report known to the authors of the employment of the AEKF, as proposed by Bizup and Brown [9], for nonlinear state estimation using bistatic Doppler.

This paper is divided into five sections, organized as follows. Section 1 introduces the topic and provides background information and context for the paper. Section 2 formulates the bistatic tracking problem, and provides an analysis of bistatic Doppler, and how it may be incorporated into multistatic target tracking using the standard and alternate EKF formulations. Simulation results are presented and analyzed in Section 3. Finally, Section 4 presents a summary of the results of the research presented in the paper and offers some conclusions and suggestions for further research.

\section{Problem Formulation}

\subsection{Target Dynamic Model}

In Cartesian coordinates, the target dynamics can be modeled in a general way as:

$$
X_{k+1}=F_{k} X_{k}+G_{k} U_{k}+J_{k} W_{k}
$$

In 2-dimensional Cartesian space, the target kinematic state vector at time $k$ is $X_{k}=\left[\begin{array}{llll}x_{k} & y_{k} & \dot{x}_{k} & \dot{y}_{k}\end{array}\right]^{T}, x_{k}$, $y_{k}$ are the position components along the $x, y$ directions; $\dot{x}_{k}, \quad \dot{y}_{k}$ are the corresponding velocity components. $F_{k} \in R^{n x n}$ is the state transition matrix; $G_{k}$ and $J_{k}$ are appropriately dimensioned coefficient matrices; $U_{k}$ is the deterministic input matrix; and $W_{k}$ is zero-mean white Gaussian process noise with variance $E\left\{w_{k} w_{k}^{T}\right\}=Q_{k}$. 


\subsection{Multistatic Sonar Measurement Equation}

Consider a multistatic sensor field consisting of multiple receivers and one or more sources, as depicted in Fig. 1. Such a field is usually processed as a set of bistatic pairs. One such bistatic pair is shown in Fig. 2. This bistatic pair consists of the source $S$ at location $\left(x_{S}, y_{S}\right)$ and the receiver $R$ at location $\left(x_{R}, y_{R}\right)$. The source-receiver baseline $L$ has length:

$L=\sqrt{\left(x_{R}-x_{S}\right)^{2}+\left(y_{R}-y_{S}\right)^{2}}$

Also shown in Fig. 2 is a target $T$ at location $\left(x_{T}, y_{T}\right)$, and bearing $\theta$ from receiver $R$. Assuming that the sound speed $c$ is constant, which is approximately true for most cases, it can be shown from geometric arguments [1] that the range $r_{R}$ of target $T$ from receiver $R$ satisfies:

$r_{R}=\frac{(c \tau)^{2}+2 L c \tau}{2[c \tau+L(1-\cos \varphi)]}$

Here $\tau$ is the elapsed time between the arrival of the direct blast at the receiver, and the arrival of the echo from target $T$ at the receiver. This quantity $\tau$ is commonly referred to as the time difference of arrival (TDOA). Finally, the angle $\varphi$ is the bearing of target $T$ with respect to the source-receiver baseline $L$, given by $\varphi=\theta-\phi$, where $\phi$ is the bearing of source $S$ relative to receiver $R$. Note that estimation of target range $r_{R}$ may be performed using (3) without knowledge of the exact pulse transmission time from source $S$, as only knowledge of the time difference $\tau$ is required.

The measured echo parameters in (3) are the TDOA $\tau$ and the target-receiver bearing $\theta$. For any TDOA $\tau$ greater than the direct blast travel time $L / c$, the locus of points on which the target may lie describes an ellipse whose two foci lie at the locations of source $S$ and receiver $R$ respectively. The intersection of this TDOA ellipse with the bearing line $\theta$ defines a unique position fix for the target location.

For target tracking purposes, there are two principal methods of employing a bistatic echo measurement vector $Z_{k}=\left(\tau_{k}, \theta_{k}\right)^{T}$, resulting from the $k$ th pulse transmission since track initialization, to update the track using the recursive Kalman formulation. In both methods, we employ the previously defined 2D-Cartesian state vector representation $X_{k}=\left(\begin{array}{llll}x_{k} & y_{k} & \dot{x}_{k} & \dot{y}_{k}\end{array}\right)^{T}$.

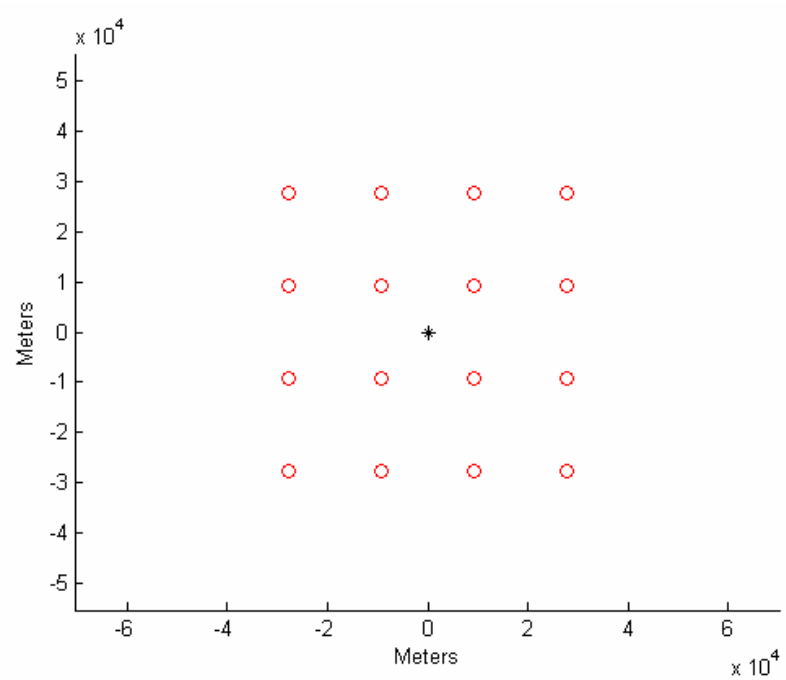

Figure 1. Typical multistatic field with a single centrally located source (star), surrounded by a rectangular grid of receivers (red circles).

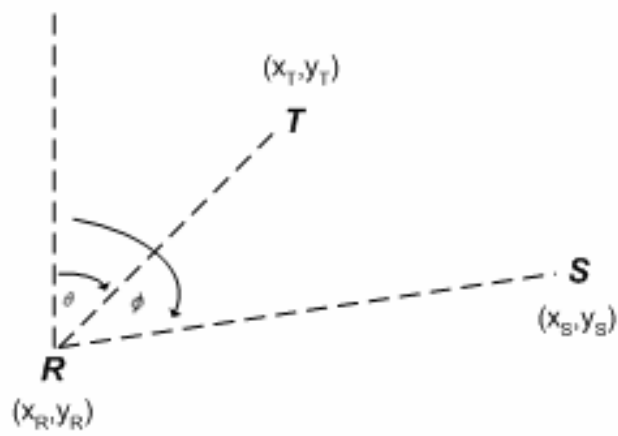

Figure 2. Bistatic Geometry.

One method is to use the Cartesian position fix defined by $Z_{k}$, and a linearized error covariance matrix for the position fix, in a linear Kalman filter. This is known as the converted measurement method. Lerro and BarShalom have presented a method for minimizing the covariance linearization errors in such an approach [15]. However, as the linear Kalman filter cannot handle highly nonlinear Doppler measurements, the approach we shall focus on in this paper is the direct approach, in which the raw measurement vector $Z_{k}$ itself is used to update the track state in an extended Kalman filter formulation. This requires linearization of the nonlinear measurement function $H$ in the exact formulation $Z_{k}=H\left(X_{k}\right)$ to 
construct the linearized measurement matrix $\hat{H}_{k}$. Writing the exact transformation matrix as $H=\left[h_{\tau} h_{\theta} h_{D}\right]^{T}$ and omitting the subscript $k$ in its elements for simplicity, we can write the first two elements of $H$ as:

$$
\begin{gathered}
h_{\tau}=\frac{1}{c}\left[r_{R}+r_{S}-L\right] \\
h_{\theta}=\tan ^{-1}\left(\frac{x_{k}-x_{R}}{y_{k}-y_{R}}\right)
\end{gathered}
$$

To construct $\hat{H}_{k}$, we require the partial derivatives of $h_{\tau}$ and $h_{\theta}$ with respect to the state variables. These are as follows:

$$
\begin{aligned}
& \frac{\partial \tau_{k}}{\partial x_{k}}=\left.\frac{1}{c}\left[\frac{\left(x-x_{S}\right)}{r_{S}}+\frac{\left(x-x_{R}\right)}{r_{R}}\right]\right|_{x=x_{k}} \\
& \frac{\partial \tau_{k}}{\partial y_{k}}=\left.\frac{1}{c}\left[\frac{\left(y-y_{S}\right)}{r_{S}}+\frac{\left(y-y_{R}\right)}{r_{R}}\right]\right|_{y=y_{k}} \\
& \frac{\partial \tau_{k}}{\partial \dot{x}_{k}}=0 \\
& \frac{\partial \tau_{k}}{\partial \dot{y}_{k}}=0 \\
& \frac{\partial \theta_{k}}{\partial x_{k}}=\left.\frac{\left(y-y_{R}\right)}{r_{R}^{2}}\right|_{y=y_{k}} \\
& \frac{\partial \theta_{k}}{\partial y_{k}}=\left.\frac{-\left(x-x_{R}\right)}{r_{R}^{2}}\right|_{x=x_{k}} \\
& \frac{\partial \theta_{k}}{\partial \dot{x}_{k}}=0 \\
& \frac{\partial \dot{y}_{k}}{\theta_{k}}
\end{aligned}
$$

Here we have employed the source-target range:

$$
r_{S}=\sqrt{\left(x_{k}-x_{S}\right)^{2}+\left(y_{k}-y_{S}\right)^{2}}
$$

Now let us consider the bistatic Doppler observation $D$. In general, the range-rate of an object at position $(x, y)$ with velocity $(\dot{x}, \dot{y})$ observed by an observer at position $\left(x_{0}, y_{0}\right)$ moving with velocity $\left(\dot{x}_{0}, \dot{y}_{0}\right)$ is given by:

$$
\begin{aligned}
\dot{r}_{0} & =\frac{\left(x-x_{0}\right)\left(\dot{x}-\dot{x}_{0}\right)+\left(y-y_{0}\right)\left(\dot{y}-\dot{y}_{0}\right)}{\sqrt{\left(x-x_{0}\right)^{2}+\left(y-y_{0}\right)^{2}}} \\
& =\frac{\left(x-x_{0}\right)\left(\dot{x}-\dot{x}_{0}\right)+\left(y-y_{0}\right)\left(\dot{y}-\dot{y}_{0}\right)}{r_{0}}
\end{aligned}
$$

Here we have denoted the range from target to observer as $r_{0} \equiv \sqrt{\left(x-x_{0}\right)^{2}+\left(y-y_{0}\right)^{2}}$.

In general, the derivatives of the range rate observed by an observer with respect to the target state variables are given by:

$$
\begin{aligned}
& H_{\dot{r}_{0}}(x) \equiv \frac{\partial \dot{r}_{0}}{\partial x}=\frac{\left(\dot{x}-\dot{x}_{0}\right)}{r_{0}}-\frac{\left(x-x_{0}\right) \dot{r}_{0}}{r_{0}^{2}} \\
& H_{\dot{r}_{0}}(y) \equiv \frac{\partial \dot{r}_{0}}{\partial y}=\frac{\left(\dot{y}-\dot{y}_{0}\right)}{r_{0}}-\frac{\left(y-y_{0}\right) \dot{r}_{0}}{r_{0}^{2}} \\
& H_{\dot{r}_{0}}(\dot{x}) \equiv \frac{\partial \dot{r}_{0}}{\partial \dot{x}}=\frac{\left(x-x_{0}\right)}{r_{0}} \\
& H_{\dot{r}_{0}}(\dot{y}) \equiv \frac{\partial \dot{r}_{0}}{\partial \dot{y}}=\frac{\left(y-y_{0}\right)}{r_{0}}
\end{aligned}
$$

Consider a continuous wave (CW) pulse of frequency $f_{0}$ and time duration $\tau_{p}$ emitted by a source at location $\left(x_{S}, y_{S}\right)$ and detected by a receiver at location $\left(x_{R}, y_{R}\right)$ via reflection from a target with state $(x, y, \dot{x}, \dot{y})$. It can be shown that the frequency $f_{\text {obs }}$ observed at the receiver is related to the source frequency $f_{0}$ by the bistatic Doppler shift $D$ :

$$
D \equiv \frac{f_{o b s}-f_{0}}{f_{0}}=-\frac{1}{c}\left(\dot{r}_{S}+\dot{r}_{R}\right)
$$

Here, $\dot{r}_{S}$ and $\dot{r}_{R}$ are the range rates of the target with respect to the source and receiver respectively, evaluated 
by substitution of the source and receiver state variables, respectively, into (8):

$$
\begin{aligned}
& \dot{r}_{S}=\frac{\left(x-x_{S}\right)\left(\dot{x}-\dot{x}_{S}\right)+\left(y-y_{S}\right)\left(\dot{y}-\dot{y}_{S}\right)}{\sqrt{\left(x-x_{S}\right)^{2}+\left(y-y_{S}\right)^{2}}} \\
& \dot{r}_{R}=\frac{\left(x-x_{R}\right)\left(\dot{x}-\dot{x}_{R}\right)+\left(y-y_{R}\right)\left(\dot{y}-\dot{y}_{R}\right)}{\sqrt{\left(x-x_{R}\right)^{2}+\left(y-y_{R}\right)^{2}}}
\end{aligned}
$$

Using the equations (9) to define the partials evaluated at the source $H_{\dot{r}_{S}}$ and receiver $H_{\dot{r}_{R}}$, the partial derivatives of bistatic Doppler shift $D$, in Hertz, are given by:

$$
\begin{aligned}
& \frac{\partial h_{D}}{\partial x}=-\frac{1}{c}\left[H_{\dot{r}_{S}}(x)+H_{\dot{r}_{R}}(x)\right] \\
& \frac{\partial h_{D}}{\partial y}=-\frac{1}{c}\left[H_{\dot{r}_{S}}(y)+H_{\dot{r}_{R}}(y)\right] \\
& \frac{\partial h_{D}}{\partial \dot{x}}=-\frac{1}{c}\left[H_{\dot{r}_{S}}(\dot{x})+H_{\dot{r}_{R}}(\dot{x})\right] \\
& \frac{\partial h_{D}}{\partial \dot{y}}=-\frac{1}{c}\left[H_{\dot{r}_{S}}(\dot{y})+H_{\dot{r}_{R}}(\dot{y})\right]
\end{aligned}
$$

Here the partials with respect to range rate observed at the source-receiver pair are obtained by substitution of the source-receiver state variables in the general range rate partial derivatives given in (9).

The complete linearized measurement matrix $\hat{H}_{k}$ is defined in terms of the partial derivatives (6) and (12) as:

$$
\hat{H}_{k} \equiv\left[\begin{array}{llll}
\frac{\partial h_{\tau}}{\partial x_{k}} & \frac{\partial h_{\tau}}{\partial y_{k}} & \frac{\partial h_{\tau}}{\partial \dot{x}_{k}} & \frac{\partial h_{\tau}}{\partial \dot{y}_{k}} \\
\frac{\partial h_{\theta}}{\partial x_{k}} & \frac{\partial h_{\theta}}{\partial y_{k}} & \frac{\partial h_{\theta}}{\partial \dot{x}_{k}} & \frac{\partial h_{\theta}}{\partial \dot{y}_{k}} \\
\frac{\partial h_{D}}{\partial x_{k}} & \frac{\partial h_{D}}{\partial y_{k}} & \frac{\partial h_{D}}{\partial \dot{x}_{k}} & \frac{\partial h_{D}}{\partial \dot{y}_{k}}
\end{array}\right]^{T}
$$

The formulation of the linearized measurement matrix $\hat{H}_{k}$ given in (13) defines the standard EKF for the multistatic tracking problem incorporating Doppler measurements.

Now we shall derive the measurement matrix $\hat{H}_{k}$ for the alternate EKF (AEKF) for bistatic Doppler. Bizup and
Brown [9] show that for the monostatic case with a single source/receiver located at the origin of the Cartesian coordinate system, the net contribution of the position state variables $x_{k}, y_{k}$ to the value of the predicted range rate at time $k$ in the standard EKF is zero. They argue, hence, for the zeroing of the partial derivatives of range rate with respect to $x_{k}, y_{k}$ in $\hat{H}_{k}$, thus defining the AEKF.

However, following their procedure of forming the dot product of the partial derivatives with the state variables and re-arranging terms with the general, bistatic form of these derivatives in (12) does not produce a zero result for the contribution of $x_{k}, y_{k}$ to the predicted Doppler observation. Rather, we find for the general case of noncollocated source and receiver, neither of which is located at the origin, and both of which are possibly moving, that the net contribution of these terms may be expressed as:

$$
\begin{array}{r}
\xi=\frac{\dot{r}_{S}}{2}\left(\frac{\left(x^{2}+y^{2}\right)+\left(x_{S}^{2}+y_{S}^{2}\right)}{r_{S}^{2}}-1\right)+ \\
\frac{\dot{r}_{R}}{2}\left(\frac{\left(x^{2}+y^{2}\right)+\left(x_{R}^{2}+y_{R}^{2}\right)}{r_{R}^{2}}-1\right)
\end{array}
$$

Note that (14) reduces to zero when the source and receiver are located at the origin, in agreement with [9]. $\xi$ is also zero when the position vectors of both source and receiver are orthogonal to the target's position vector, which will rarely occur in practice; $\xi$ will otherwise be nonzero for moving targets.

Clearly we cannot have both the source and the receiver located at the origin in a bistatic system. We may chose, however, to locate one of these at the origin; the usual choice, for tracking purposes, being to place the receiver at the origin. Following this practice, only the source term in (14) remains nonzero. We therefore conclude that, while we cannot zero the entire partial derivatives of Doppler with respect to $x_{k}, y_{k}$ in $\hat{H}_{k}$, we may zero the components of these partial derivatives in the receiver. The AEKF for bistatic Doppler, then, is defined by replacing (12a) and (12b) with the following:

$$
\begin{aligned}
& \frac{\partial h_{D}}{\partial x}=-\frac{1}{c}\left[H_{\dot{r}_{S}}(x)\right] \\
& \frac{\partial h_{D}}{\partial y}=-\frac{1}{c}\left[H_{\dot{r}_{S}}(y)\right]
\end{aligned}
$$




\section{Simulation Results}

In order to compare the performance of the standard EKF and AEKF formulations in a multistatic tracking context, a number of simulations were performed using the stationary multistatic field depicted in Fig. 1. These simulations were conducted using one of two sets of uncorrelated zero-mean white Gaussian measurement errors: small-error simulations with $\sigma_{\tau}=0.01 \mathrm{~s}$, $\sigma_{\theta}=0.5^{0}$, and large-error simulations with $\sigma_{\tau}=0.1 s, \sigma_{\theta}=5^{0}$. The large errors are typical of some current and planned fielded systems for underwater tracking. The Doppler measurement noise in all cases is modeled as $\sigma_{D}=0$; that is, the Doppler measurements are ideal. Effects of non-ideal Doppler observations are considered later. Each simulation comprised a set of fifty 1 -second CW pulses emitted at a rate of one per minute at a frequency of $f_{0}=1800 \mathrm{~Hz}$. Each target was modeled using a strongly aspect-dependent "glinty" reflectance model such that generally each receiver was only in contact with the target for a small number of consecutive pings when it was in the correct specular reflection geometry with the target and the source. The "average" probability of detection was approximately $P_{D}=0.5$. There were no false alarms. Velocity estimation data was collected over 50 Monte Carlo trials for each configuration under test. The performance metric that was collected was the RMS velocity error, computed in terms of the mean square $\mathrm{x}$ - and y-velocity errors $\bar{\sigma}_{x}^{2}$ and $\bar{\sigma}_{y}^{2}$ as:

$$
\mathcal{E}_{R M S}=\sqrt{\bar{\sigma}_{x}^{2}+\bar{\sigma}_{y}^{2}}
$$

The tracking system used for all trials was a distributed architecture data fusion system design with each receiver defining a local node, and a global node receiving and fusing tracks reported by the local nodes. The fused tracks produced at the global node constitute the output of the tracking system. The local trackers were implementations of $\mathrm{N}$-scan sliding window multiple hypothesis tracking (MHT) with a hypothesis depth of $\mathrm{N}=1$ previous scan [16]. Track fusion was implemented in the global node using the information fusion method [17]. The local tracker nodes employed a single nearly constant velocity (NCV) target motion model in trials with transiting targets, and a fixed structure interacting multiple model (IMM) nonlinear filter [18] incorporating the NCV motion model for transiting targets and a second NCV model with increased bandwidth, for trials with maneuvering targets.

\subsection{Monostatic Simulations}

To set a performance baseline, a number of preliminary simulations were conducted using a single monostatic pair. These simulations showed that the standard EKF performs reasonably well with both small and large measurement errors against both transiting (at nearly constant velocity) and maneuvering targets in a monostatic system. The ten maneuvering targets had trajectories as shown in Fig. 3, where the passage of time is indicated by the colour of each target track progressing from blue to green. In these simulations, the processing of Doppler observations using the standard EKF produced a small but measurable improvement in the velocity estimates of transiting targets. Noticeably greater improvements were observed for maneuvering targets when the errors were small, but the improvements were negligible for maneuvering targets when the errors were large. Including Doppler observations had a beneficial effect, or at worst a neutral effect, on EKF performance in the monostatic simulations.

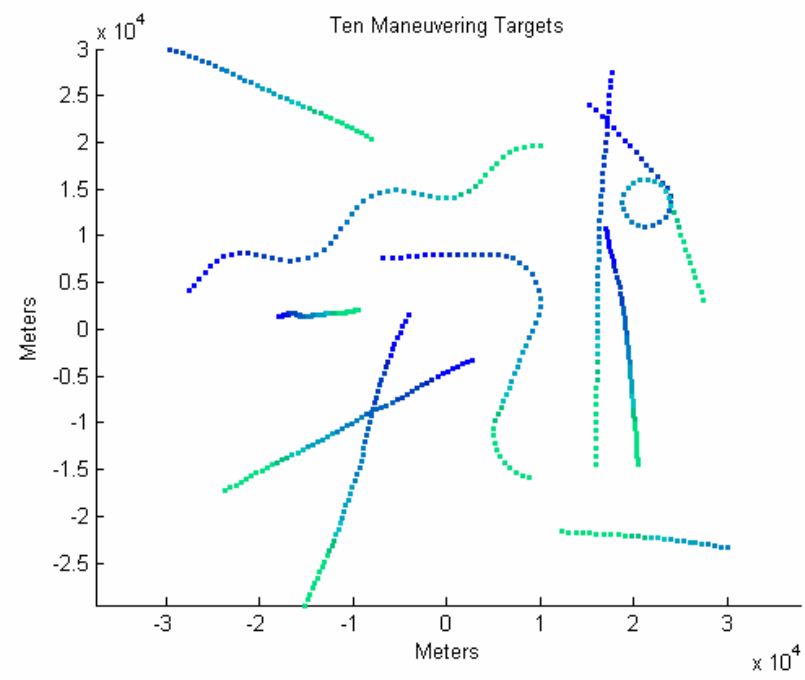

Figure 3. Maneuvering target simulation.

\subsection{Multistatic Simulations}

The performance trend observed in the monostatic trials continues in the multistatic trials with small measurement errors, as shown in Fig. 4. Here the EKF with Doppler is shown to have significantly better velocity estimation than either the EKF without Doppler, or the AEKF. However, the situation changes dramatically in the multistatic case when the measurement errors become realistically large, as shown in Fig. 5. Here we see that velocity estimation errors can actually be larger with the EKF with Doppler than when Doppler is not employed. The AEKF, on the other hand, is shown to produce velocity estimation errors significantly lower than the EKF with or without Doppler. 


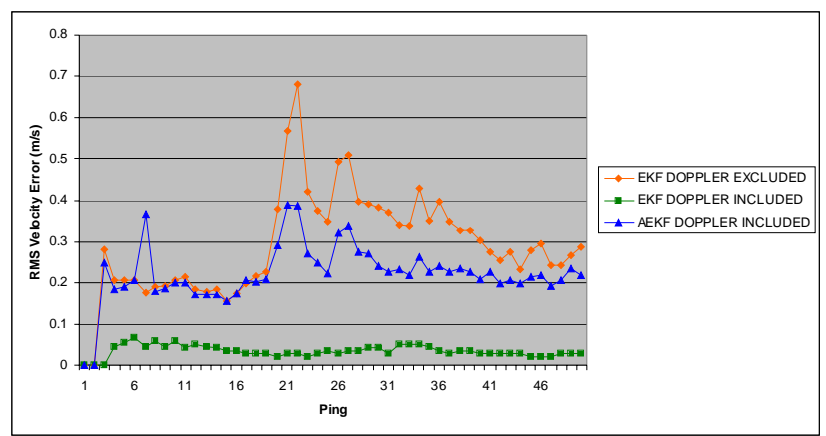

Figure 4. Multistatic simulation results with small measurement errors, averaged over 10 trajectories.

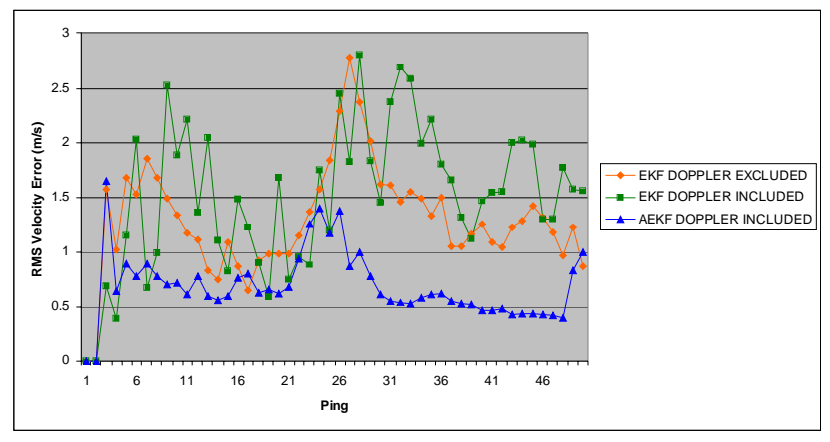

Figure 5. Multistatic simulation results with large measurement errors, averaged over 10 trajectories.

The simulations reported so far have used ideal Doppler measurements. In practical systems, the ability to measure frequency is limited. This limited frequency measurement ability may be modeled as a quantization of frequency measurements. To quantify the potentially degrading effects of frequency quantization on the benefits of tracking with Doppler observations, a final series of trials was conducted using only the bistatic AEKF, with Doppler observations computed from frequency observations quantized to $0.25 \mathrm{~Hz}, 0.50 \mathrm{~Hz}$, and $1.0 \mathrm{~Hz}$. The results, shown in Fig. 6, reveal that the degrading effect, if any, is very minor for all three quantizations, suggesting that the bistatic AEKF should work well in most practical systems.

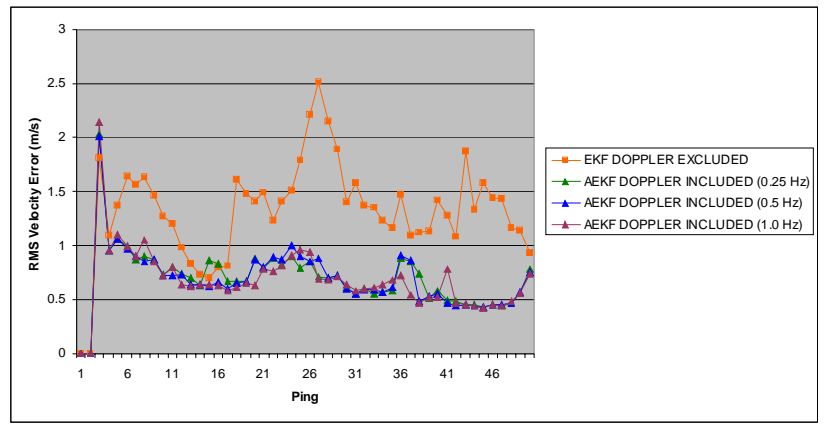

Figure 6. Multistatic simulation results with large errors and quantized frequency observations.

\section{Summary and Conclusions}

This paper has considered the exploitation of bistatic Doppler measurements in a multistatic sonar tracking context. It was found through simulation that, while the standard EKF works reasonably well with Doppler measurements, in the sense of producing better target velocity estimates than when Doppler measurements are not processed, for monostatic situations, and multistatic situations where the measurement errors are small, it does not work well in multistatic cases where measurement errors are large. In fact, a significant degradation in state estimation accuracy is observed in the latter case. The observed effects of processing Doppler measurements in this case were therefore negative.

It was found, through further simulation of the multistatic maneuvering target case with realistic, large measurement errors, that usefully positive velocity estimate improvements could be obtained by replacing the standard EKF with the alternate EKF (AEKF) proposed by Bizup and Brown [9], modified for bistatic measurement vectors. We conclude therefore, that it may be possible to improve target velocity estimation accuracy in real multistatic applications by processing Doppler observations with the bistatic AEKF. Our results indicate that this conclusion applies even in the presence of quantized frequency observations as produced by real systems. But the natural phenomena behind multistatics are complex and the simulation results will not necessarily be realized in fielded systems.

There may be alternative, perhaps superior, methods for incorporating Doppler observations into state estimation. Several papers have reported improved state estimation performance when range-rate, or Doppler, measurements are processed in a specific order using a sequential EKF (SEKF) [16]-[18], or a sequential Unscented Kalman filter (SUKF) [13], instead of the standard EKF. Also, we have limited our consideration here to $\mathrm{CW}$ pulses, for which the measurement errors in range, TDOA, and Doppler are uncorrelated. Extension of the analysis to other pulse types, for example linear frequency modulation (LFM) pulses, requires consideration of the correlation between range and range-rate measurement errors arising from the use of such pulse types [10]-[13].

\section{References}

[1] Harry Cox, "Fundamentals of Bistatic Active Sonar," in Underwater Acoustic Signal Processing (Y.T. Chan, ed.), Kluwer Academic Publishers, 1989.

Stefano Coraluppi and Doug Grimmett, "Multistatic Sonar Tracking," Proceedings of the SPIE Conference on Signal Processing, Sensor 
Fusion, and Target Recognition XII, Orlando FL, USA, 2003.

Pascal De Theije, L. Kester and J. Bergmans, "Application of the M6T tracker to simulated and experimental multistatic sonar data," Proceedings of the Ninth International Conference on Information Fusion, Florence, Italy, 2006.

Brian La Cour, "Bayesian Multistatic Tracking: Results on Simulated Data from the Multistatic Tracking Working Group," Proceedings of the Ninth International Conference on Information Fusion, Florence, Italy, 2006.

Peter Willett, P. and Stefano Coraluppi, "MLPDA and MLMHT applied to MSTWG data," Proceedings of the Ninth International Conference on Information Fusion, Florence, Italy, 2006.

[6] Christian Hempel, "Probabilistic MultiHypothesis Tracking for Distributed Active Sonar," Proceedings of the Ninth International Conference on Information Fusion, Florence, Italy, 2006.

[7] Alfonso Farina, and F.A. Studer, Radar Data Processing, Volume 1: Introduction and Tracking, Volume 2: Advanced Topics and Applications, Research Studies Press, Letchworth, 1985.

[8] H. Kameda, S. Tsujimichi and Y. Kosuge, "Target tracking under dense environment using range rate measurements," Proc. 37th SICE Annual Conference, Chiba, Japan, 1998.

[9] D.F. Bizup, and D.E. Brown, "The OverExtended Kalman Filter - Don't Use It!," Proceedings of the Sixth International Conference on Information Fusion, Vol. 1, Cairns, Australia, 2003.

[10] Yaakov Bar-Shalom, "Negative correlation and optimal tracking with Doppler measurements," IEEE Trans. Aerospace and Electronic Systems, Vol. 37, No. 3, March 2001.

[11] J. Wang, P. He and T. Long, "Use of the radial velocity measurement in target tracking," IEEE Trans. Aerospace and Electronic Systems, Vol. 39, No. 2, February 2003.
Z. Duan, C. Han and X.R. Li, "Sequential nonlinear tracking filter with range-rate measurements in spherical coordinates," Proceedings of the Seventh International
Conference on Information Fusion, Stockholm, Sweden, 2004.

Z. Duan, X.R. Li, C. Han and H. Zhi, "Sequential Unscented Kalman Filter for radar target tracking with range rate measurements," Proceedings of the Eighth International Conference on Information Fusion, Philadelphia PA, USA, 2005.

[14] Doug Grimmett, "Multi-sensor placement to exploit complementary properties of diverse sonar waveforms," Proceedings of the Ninth International Conference on Information Fusion, Florence, Italy, 2006.

D. Lerro and Y. Bar-Shalom, "Tracking with Debiased Consistent Converted Measurements versus EKF," IEEE Trans. Aerospace and Electronic Systems, Vol. 29, No. 3, March 1993.

[16] R.L. Popp, K.R. Pattipati and Y. Bar-Shalom, "m-best S-D Assignment Algorithm with Application to Multitarget Tracking," IEEE Trans. Aerospace and Electronic Systems, Vol. 37, No. 1, January 2001.

[17] K.C. Chang, T. Zhi, and R.K. Saha, "Performance Evaluation of Track Fusion with Information Matrix Filter," IEEE Trans. Aerospace and Electronic Systems, Vol. 38, No. 2, April 2002.

[18] X.R. Li and V.P. Jilkov, "Survey of Maneuvering Target Tracking Part V: Multiple Model Methods," IEEE Trans. Aerospace and Electronic Systems, Vol. 41, No. 4, October 2005.

[19] K.S. Miller and D.M. Leskiw, "Nonlinear Estimation with Radar Observations," IEEE Trans. Aerospace and Electronic Systems, Vol. 18, No. 2, February 1982.

[20] S.E. Park and J.G. Lee, "Design of a Practical Tracking Algorithm with Radar Measurements," IEEE Trans. Aerospace and Electronic Systems, Vol. 34, No. 4, April 1998.

[21] S.E. Park and J.G. Lee, "Improved Kalman Filter Design for Three-Dimensional Radar Tracking," IEEE Trans. Aerospace and Electronic Systems, Vol. 37, No. 2, February 2001. 